CAHIERS DE

NARRATOLOGIE

\section{Cahiers de Narratologie}

Analyse et théorie narratives

$20 \mid 2011$

Voix off et narration cinématographique

\title{
Les frontières sonores de la voix-over
}

Étude de cas avec le film De Beaux Lendemains, d'Atom Egoyan (1997)

Séverine Abhervé

\section{(2) OpenEdition}

Journals

Édition électronique

URL : http://journals.openedition.org/narratologie/6317

DOI : $10.4000 /$ narratologie.6317

ISSN : 1765-307X

Éditeur

LIRCES

Référence électronique

Séverine Abhervé, «Les frontières sonores de la voix-over », Cahiers de Narratologie [En ligne], 20 | 2011, mis en ligne le 26 août 2011, consulté le 01 mai 2019. URL : http://journals.openedition.org/

narratologie/6317; DOI : 10.4000/narratologie.6317

Ce document a été généré automatiquement le 1 mai 2019.

Article L.111-1 du Code de la propriété intellectuelle. 


\title{
Les frontières sonores de la voix-
}

\section{over}

\author{
Étude de cas avec le film De Beaux Lendemains, d'Atom Egoyan (1997)
}

\section{Séverine Abhervé}

1 À la sortie du film De Beaux Lendemains ${ }^{1}$, le critique Didier Péron releva l'importance de la bande sonore dans le processus narratif : «[...] Le motif sonore, ou vocal, traverse [...] le film et devient vite essentiel [...]. C'est [...] bien le charme de la musique qui mène la danse et le son qui guide nos pas sur la plate-forme risquée du film, patinoire esthétique pour débâcles intérieures²."

2 Ce motif vocal dont il est question, c'est bien l'argument que nous nous proposons de développer ici : étudier les multiples formes sonores que peut revêtir la voix over dans l'audiovisuel et en analyser la portée sur la narration cinématographique. Pour ce faire, nous travaillerons sur plusieurs extraits du film, en nous attachant particulièrement à la voix over du personnage de Nicole.

Précisons la terminologie choisie : nous sollicitons le terme voix over selon la distinction de Sarah Kozloff (1988) commune à celle de Laurent Jullier (1995) et de Jean Châteauvert auprès de qui nous empruntons la définition suivante (1996: p. 210) : « la voix over n'est pas accessible aux personnages du monde visualisé, le discours n'étant accessible qu'à des personnages participant d'un monde enchâssant, voire aux seuls spectateurs. Le discours en voix over pourra être indépendant du monde visualisé, se présenter comme un commentaire à propos de ce monde ou en assurer explicitement la narration. »

4 La voix de Nicole subit, tout au long du récit, des modifications touchant à son statut narratif et à la responsabilité de son énoncé. Son traitement est varié : cette voix est présentée à travers celle du personnage parlant ou lisant, ou encore celle de la musicienne chantant. Elle est enfin sollicitée par le procédé de la voix over. Or, les frontières entre ces différents traitements vocaux s'effacent progressivement. Le personnage de Nicole nous amène donc à repenser les frontières sonores de la voix over.

5 Par le mot " frontière ", nous faisons allusion au classement des sons au sein de la bande sonore. Laurent Jullier (2006: pp. 43-44) place sur deux axes symboliques les «sons 
organisés " (axe du verbal, axe du musical) qui représentent les deux premières catégories. Une troisième catégorie est celle du bruit : substance sonore inorganisée ; la quatrième représente le silence. Le passage d'une catégorie sonore à une autre se réalise au sein d'un continuum sonore plus ou moins bien « domestiqué ».

Selon Laurent Jullier, le principal intérêt dans l'étude de la bande son est de comprendre l'utilisation de ces différents systèmes sonores, qui peuvent, parfois, exister au sein de plusieurs catégories. La chanson est donnée en exemple comme un "son hybride" sollicitant autant la catégorie de la parole que celle de la musique. Nous pouvons donc reformuler cet exemple en expliquant que la voix, rencontrant la musique dans le continuum sonore du film, traverse la frontière entre ces deux catégories. La voix over, symboliquement placée sur l'axe verbal, nous interroge sur sa capacité à s'hybrider au contact des autres catégories sonores.

7 Si la théorie des sons nous a plus souvent donné à penser ses propres frontières par rapport à l'image - son in, son hors-champ ou son off selon Michel Chion (2003: p. 233) -, à travers cette étude, il s'agira de détailler le traitement de la voix over selon ses propres frontières sonores internes : en l'occurrence, dans De Beaux Lendemains, son déplacement entre les axes de la parole et de la musique.

8 Afin d'étudier cette structure particulière du récit filmique, commençons à observer sa source.

\section{L'adaptation cinématographique du roman éponyme}

9 Avec son septième film, le metteur en scène choisit pour la première fois d'adapter un roman. De Beaux Lendemains (The Sweet Hereafter, 1997) est le titre éponyme du livre de Russell Banks ${ }^{3}$. Un fait divers en est à l'origine : en 1989, un accident de bus scolaire dans le Sud du Texas cause la mort de vingt et un jeunes collégiens. Des dizaines d'avocats se présentent aux familles concernées par ce drame pour engager un procès. Russell Banks conserve ces éléments, il transpose l'action dans la ville fictionnelle de Sam Dent qu'il situe dans l'État de New York et construit ses propres personnages: Dolores Driscoll, le chauffeur de bus ; Billy Ansel, le garagiste et père des jumeaux Jessica et Mason décédés dans l'accident ; Mitchell Stephens, un avocat qui se présente aux familles parce qu'il a lui aussi failli perdre sa fille Zoé ; et Nicole Burnell, une jeune sportive subissant un inceste de la part de son père. Rescapée de l'accident, elle est désormais paralysée. Le roman de Russell Banks est construit autour de ces quatre personnages qui expriment, chapitre après chapitre, leur nouvelle vie à la suite de l'accident. Nous avons donc quatre narrateurs : Dolorès, Billy, Mitchell Stephens et Nicole. Atom Egoyan, tout en restant très proche du roman, décide de le mettre en images avec quelques aménagements narratifs.

C'est ainsi que Mitchell Stephens arrive un jour à Sam Dent, petite communauté canadienne, pour proposer ses services aux familles endeuillées. Il tente de les convaincre de s'engager dans un procès afin d'obtenir réparation pour la perte de leur enfant, ce que fait la famille Burnell. Leur fille, Nicole, jeune musicienne pleine de talent, a survécu au drame mais elle est dorénavant invalide. Ses parents souhaitent obtenir une compensation financière pour les soins dont elle va avoir besoin. $\mathrm{Si}$, dans un premier temps, Nicole accepte le choix de sa famille, elle décidera finalement de faire une fausse déposition devant les problèmes moraux que pose le procès à certains habitants de Sam Dent, et notamment à Billy Ansel dont elle gardait souvent les enfants. Grâce à ce 
mensonge, elle met fin au procès et, par la même occasion, punit son père de leur relation incestueuse. Après le départ de Mitchell, les villageois de Sam Dent essayent peu à peu de retrouver une vie normale.

11 Si nous comparons les deux versions filmique et romanesque des Beaux Lendemains, nous pouvons isoler quelques modifications effectuées par le réalisateur: tout d'abord, la structure du récit cinématographique, loin de respecter la chronologie des faits, nous perd dans les différentes temporalités : avant l'accident, pendant et après celui-ci. La structure narrative est totalement déconstruite, bien au-delà de simples inserts et flashbacks. De plus, l'action est déplacée des États-Unis au Canada; Nicole devient musicienne au lieu de pom-pom girl et le livre qu'elle lit aux jumeaux de Billy n'est plus Babar et les éléphants, mais Le Joueur de flûte de Hamelin de Robert Browning ${ }^{4}$. Ce dernier est particulièrement intéressant puisqu'il participe à plusieurs reprises à la mise en scène de la voix over.

12 Les divergences entre le récit filmique et le roman sont principalement liées à la musique. Nicole comme le Joueur de flûte représentent un musicien.

\section{Les modèles théoriques des chansons dans les films} l'étude de la chanson et son rapprochement avec la voix over (1995: p. 153) : «Les sons que l'on identifie comme des mots prononcés par des voix humaines sont les objets sonores que nous savons le mieux écouter ». Ici donc, la parole chantée, ainsi que la voix over sont de nature à être entendues, comprises et étudiées. (1998: p. 282) : "La chanson est cet élément simple et caractéristique, symbole d'un destin enfermé en quelques notes et en quelques mots, qui peut se promener dans tout un film, siffloté, chantonné, entonné avec ou sans paroles, dans l'écran ou depuis la fosse. Elle incarne, au cinéma, le principe même de la circulation ». Cette caractéristique peut se rapprocher de la voix over que l'on a tendance à définir comme omnipotente.

Pour finir, Jacques Aumont (1999 : p. 179) s'est intéressé à la mise en scène des chansons, qu'il situe entre deux extrémités variables : une limite dramatique (son discours offre des clefs de lecture sur la narration ou sur les personnages, elle est impliquée dans la narration filmique) et une limite musicale (elle est donnée à entendre pour elle-même, sans avoir à faire avancer le drame). Nous isolerons donc, dans le film, les chansons sollicitées au plus proche de leur limite dramatique pour étudier comparativement leurs différentes fonctions à celles de la voix over. 


\section{Les modèles théoriques des voix over dans les films}

Jean-Louis Alibert (2008, pp. 76-77), inspiré par les travaux de Michel Chion, a énoncé les principales caractéristiques de la voix over. Elle peut être omnisciente puisqu'elle sait généralement ce qui précède ou suit l'action; omnipotente, dans le sens où elle représente la « voix du pouvoir » en étant partout; puis, acousmatique lorsqu'elle n'est pas la voix d'un personnage incarné à l'écran.

Ces caractéristiques se rapprochent, en partie, de celles énoncées par Laurent Jullier (2006 : pp. 60-61) : " [La voix over] nous explique les images, les [lit] à notre place ou nous [souffle] à l'oreille, une manière de [la] comprendre qui nous convainc presque toujours... " Elle informe sur le lieu, l'époque de l'action, communique les noms des personnages et renseigne sur les mondes intérieurs. Enfin, « la prise de pouvoir de la voix off sur la bande-image se fait essentiellement par le biais de deux fonctions »: la dénominative (elle qualifie et nomme les objets ou personnes visualisés à l'écran) ou la fonction de génie (elle matérialise sur la bande-image les choses ou les gens qu'elle nomme).

21 Pour notre part, ce sont principalement les écrits de Jean Châteauvert (1996) que nous allons exposer puisqu'il développe autant de théories complémentaires que celles d'Oswald Ducrot, de François Jost ou encore de Gérard Genette (1972). Selon lui, le narrateur verbal qui s'exprime à travers la voix over dans le film participe à sa narration en tant que sujet embrayeur du monde enchâssé (il donne accès aux informations relatives au monde cité). Celui-ci oriente la lecture du spectateur-auditeur sur le monde " audiovisualisé ». Il subjectivise le récit filmique de son point de vue et introduit la possibilité d'ouvrir la narration à un double récit (entre ses propos et l'image).

Jean Châteauvert nous poussera donc à nous intéresser, dans l'analyse des extraits du film, à quatre principales interrogations :

1. à qui appartient la responsabilité de l'énoncé de la voix over (selon Ducrot)?

2. quel est le statut du narrateur (selon Genette)?

3. quelle est la fonction de la voix over?

4. qui est le destinataire du discours énoncé ?

\section{Analyse des interventions de la voix over de Nicole dans le film}

Au cours d'un entretien, Atom Egoyan, donnait son point de vue sur l'utilisation de la voix over dans son film :

[...] le scénario [...] recourait constamment [à la voix over], ce qui est inhabituel chez moi car je suis très méfiant à l'égard de ce procédé. Mais, quand j'ai regardé le boutà-bout, je me suis rendu compte que les mensonges et les soupçons, quand ils sont exprimés par la voix over, deviennent très banals. [...] Nous avions tourné des gros plans de Nicole qui intervenaient au moment où sa voix over se faisait entendre, mais, dès que nous l'avons supprimée, cela a donné une bien plus grande intensité à son regard. Quand on entendait ce qu'elle pensait, tout devenait beaucoup plus ordinaire $^{5}$ 
Une étude attentive du scénario nous a permis de vérifier les propos du réalisateur. De nombreuses interventions en voix over ont été supprimées du récit filmique et parfois remplacées au profit de la chanson ou encore d'une lecture du conte du Joueur de Flûte. Il devient alors tout à fait pertinent d'étudier les interventions chantées et lues de Nicole au même titre que celles exprimées par la voix over. C'est en ce sens que nous nous interrogerons sur les frontières sonores de sa voix : peut-elle se décliner sur l'axe verbal comme une voix over parlée et une voix over lue, puis traverser sa frontière avec l'axe musical pour devenir une voix over chantée?

\section{Extrait 1 (de $0: 05: 36$ à $0: 06: 48$, avant l'accident de bus)}

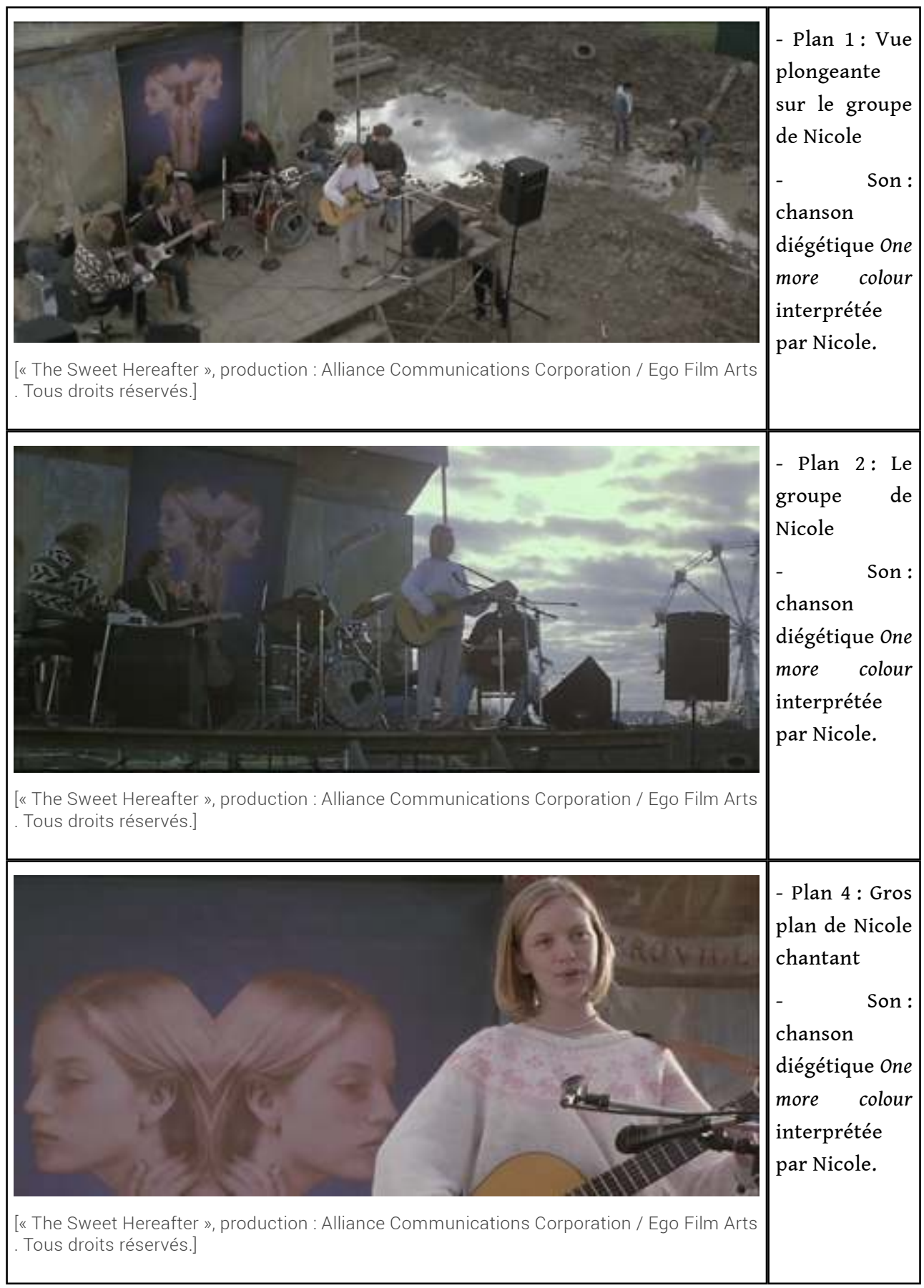




\section{Extrait 2 (de $0: 22: 49$ à $0: 23: 33$, avant l'accident de bus)}

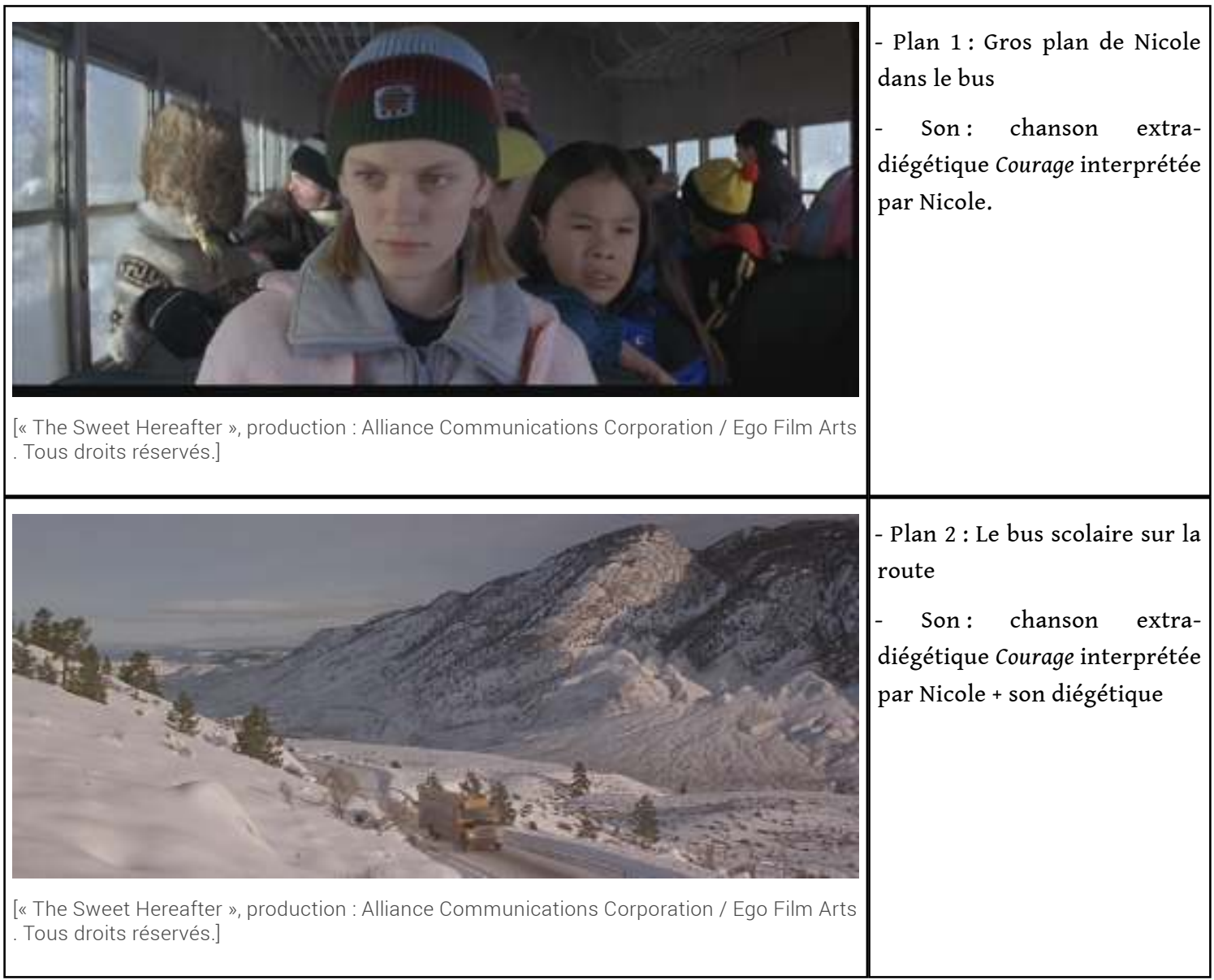


À ce stade du film, le spectateur sait qu'un accident de bus scolaire va coûter la vie à la plupart des enfants du village de Sam Dent. Nicole est ici précisément dans le bus scolaire. Une nouvelle chanson de son répertoire (on identifie sa voix) est diffusée à l'image. Pour autant, Nicole n'a pas de walkman sur les oreilles. On en déduit donc aisément que c'est une musique extra-diégétique ou encore une musique de fosse. Comme la voix chantée est celle du personnage à l'écran, tentons à présent une première analyse d'une voix over chantée.

Cette scène a été réalisée différemment par rapport au scénario qui devait nous donner à voir les jumeaux Ansel à la place de Nicole. Ce changement justifie d'autant plus l'analyse. Quel est le bénéfice de cette nouvelle proposition filmique pour le récit?

Le titre est Courage des Tragically Hip (1992), un groupe canadien extrêmement populaire. Ici encore, la chanson originale est dénaturée par sa réorchestration avec les mêmes instruments anciens du groupe de Nicole. Moins énergique, la reprise semble être devenue une ballade. La corrélation audio-visuelle interroge. Diffusée sur un court extrait ( $0: 15$ - $0: 52 / 4: 22)$, la première phrase retentit sur un gros plan du visage de Nicole : Watch the band through a bunch of dancers ${ }^{7}$. Les phrases suivantes accompagnent le second plan de cette scène : le bus scolaire sur une route longe les montagnes. Il est suivi d'une voiture, celle de Billy Ansel. Quickly, follow the unknown with something more familiar. Quickly something familiar. Courage, my word it didn't come it doesn't matter'.

2 La première phrase peut interpeller : «Courage, mon mot » est prononcée par Nicole, elle semble se parler à elle-même. Diffusée sur son visage, elle s'adresse néanmoins au spectateur-auditeur afin de lui faire passer l'idée selon laquelle Nicole va avoir besoin de « courage » pour surmonter l'accident à venir. Nous sommes ici dans une anticipation d'une action future. À ce stade du récit, aucun autre élément ne peut être éloquent, mais le lien avec l'accident apparaît évident face au plan du bus accompagné de la seconde phrase : « suis l'inconnu avec quelque chose de plus familier ».

Du point de vue de la responsabilité des propos, Nicole est énonciatrice puisqu'elle est l'interprète en tant que chanteuse et non son auteur. Étant par ailleurs à l'écran au même moment, elle devient un narrateur autodiégétique, mais comme sa voix ne semble pas être intérieure (puisqu'elle se poursuit sur le plan suivant), elle n'a pas conscience qu'elle est ici investie directement d'un rôle précis par la chanson. Le fait que Courage semble annoncer un événement futur, on peut lui conférer, à l'instar d'une voix over, la fonction de génie qui consiste à anticiper sur l'action. Pour cette raison, il apparaît tout à fait dommageable pour la narration filmique que les sous-titres de la chanson soient inexistants alors qu'elle semble omnisciente. Le spectateur, s'il devient également un auditeur attentif, peut commencer à entrer, par le biais du son, dans un double récit qu'il ne mesure pas encore. Tout comme Nicole, il se prépare à « suivre l'inconnu de façon plus familière ». 
Extrait 3 (de $0: 40: 56$ à $0: 41: 50$, avant l'accident de bus)

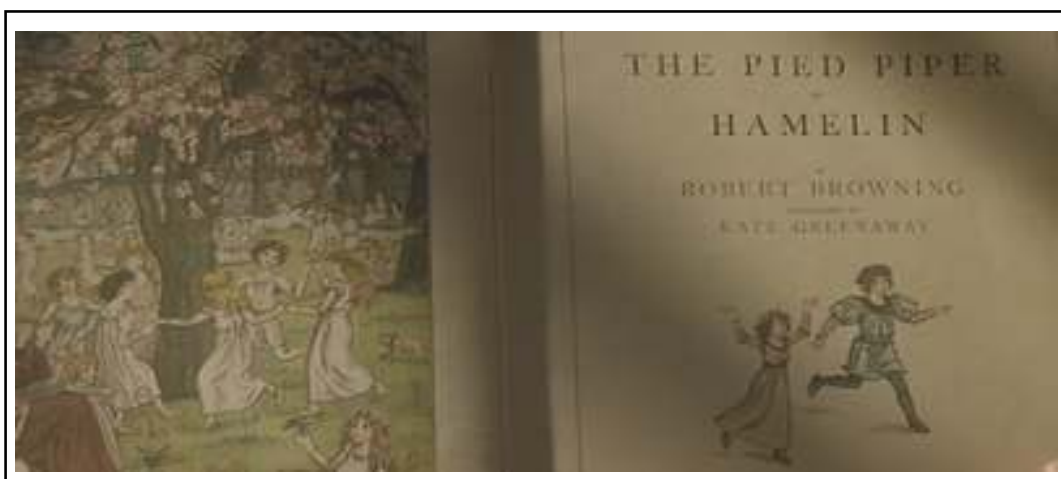

- Plan 1: Gros plan du livre Joueur Flûte.

- Son in: Nicole lit le conte.

[« The Sweet Hereafter », production : Alliance Communications Corporation / Ego Film Arts Tous droits réservés.]

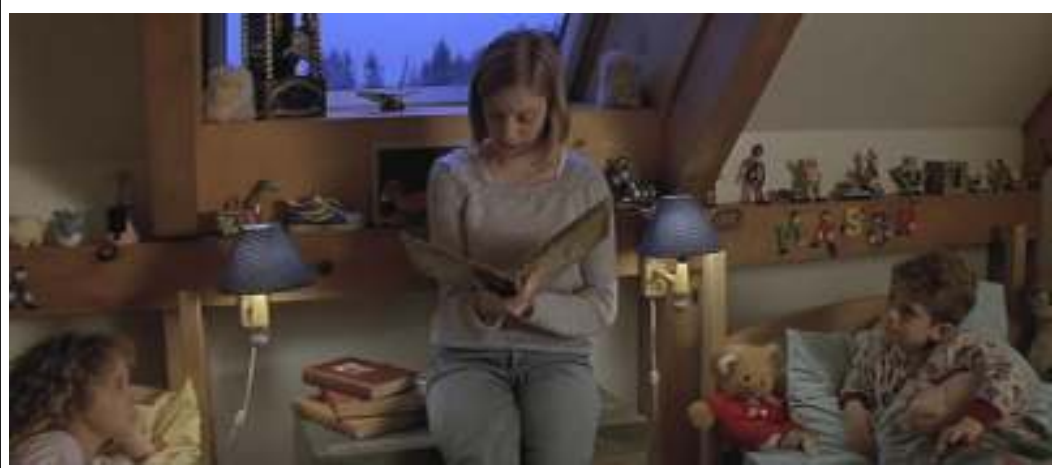
ses derniers. - Son in: Nicole lit le conte.

[«The Sweet Hereafter », production : Alliance Communications Corporation / Ego Film Arts Tous droits réservés.]

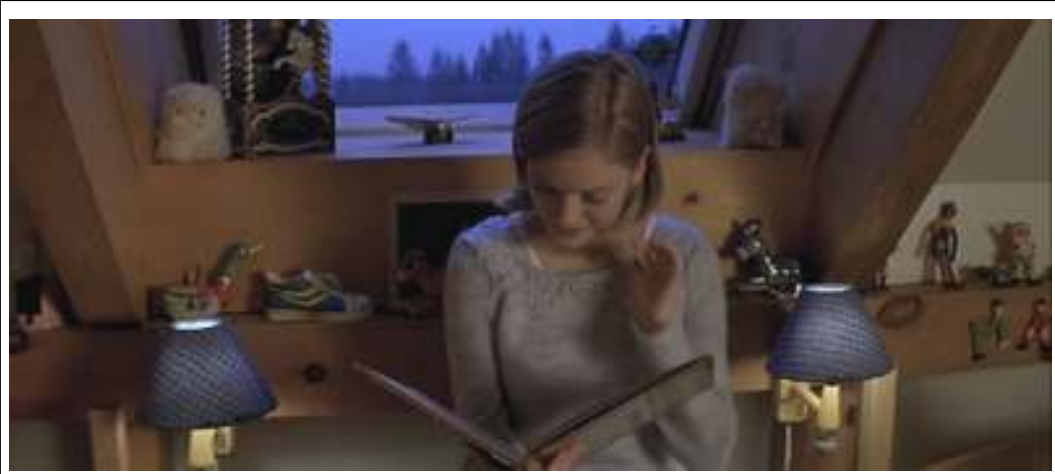

Travelling vers Nicole et le livre - Son in : Nicole lit le conte.

[«The Sweet Hereafter », production : Alliance Communications Corporation / Ego Film Arts Tous droits réservés.] 


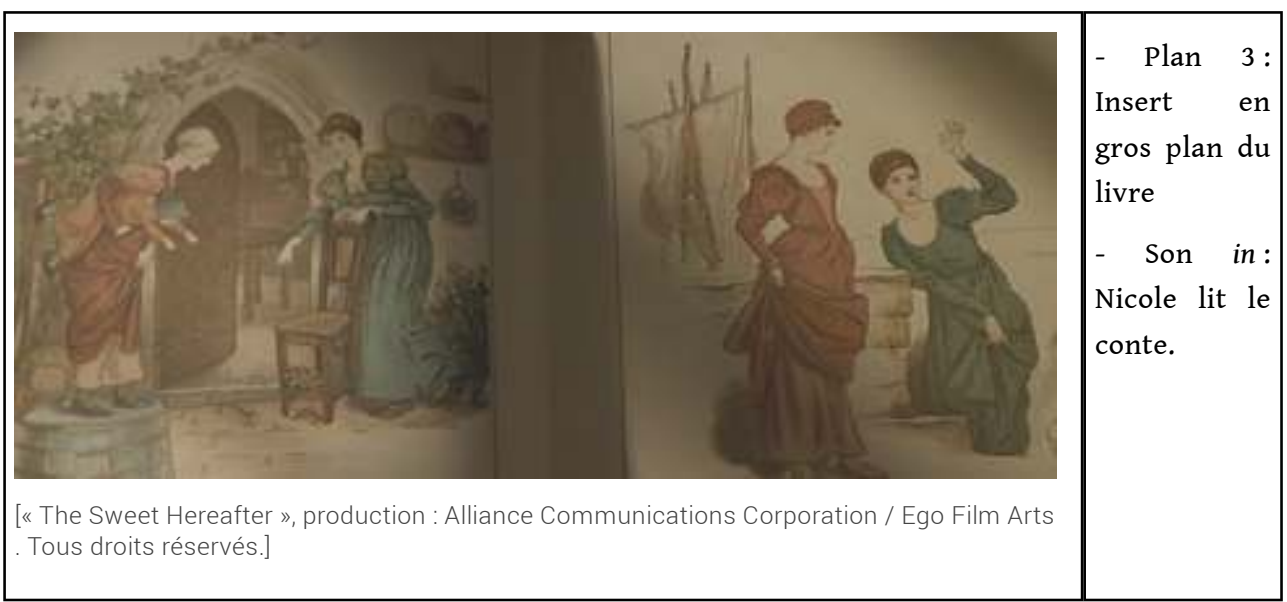

Cet extrait introduit le procédé de la voix over lue. Nicole est chez Billy Ansel qui est sorti ce soir-là. Elle garde ses jumeaux, Jessica et Mason, et leur lit, au moment du coucher, le conte du Joueur de Flûte. La scène démarre sur un gros plan du livre ouvert à la page titre. La voix de Nicole est diégétique (in), mais comme pour l'extrait 1 , cette scène est importante pour la suite du récit filmique, raison pour laquelle nous l'étudions brièvement.

Nicole lit les premières pages du conte. La caméra entame un travelling avant qui finit par l'encadrer seule avec le livre ouvert. Mason interrompt la lecture en demandant à Nicole de bien vouloir s'asseoir à côté d'elle dans le bus, le lendemain. Le spectateur ne le sait pas encore, ce sera précisément le jour de l'accident. La lecture entamée présente le récit dans la ville médiévale d'Hamelin. Ici, le récit n'a pas d'autres fonctions, à première vue, que d'être présenté pour lui-même.

Nicole, en position de lectrice, lit le conte, c'est un narrateur intradiégétique qui nous relate le récit enchâssé du Joueur de flûte. Rappelons-nous que ce conte a été introduit volontairement par le réalisateur dans le film. On peut donc imaginer que le destinataire, à part Jessica et Mason, est surtout le spectateur-auditeur, ce qui est d'ailleurs justifié par les gros plans des pages du conte. Ce dernier doit visualiser le conte pour commencer à le matérialiser, à le reconnaître. Cette scène est une introduction au double récit ouvert sur le Joueur de flûte dans la diégèse.

L'extrait suivant nous en montre les enjeux. 


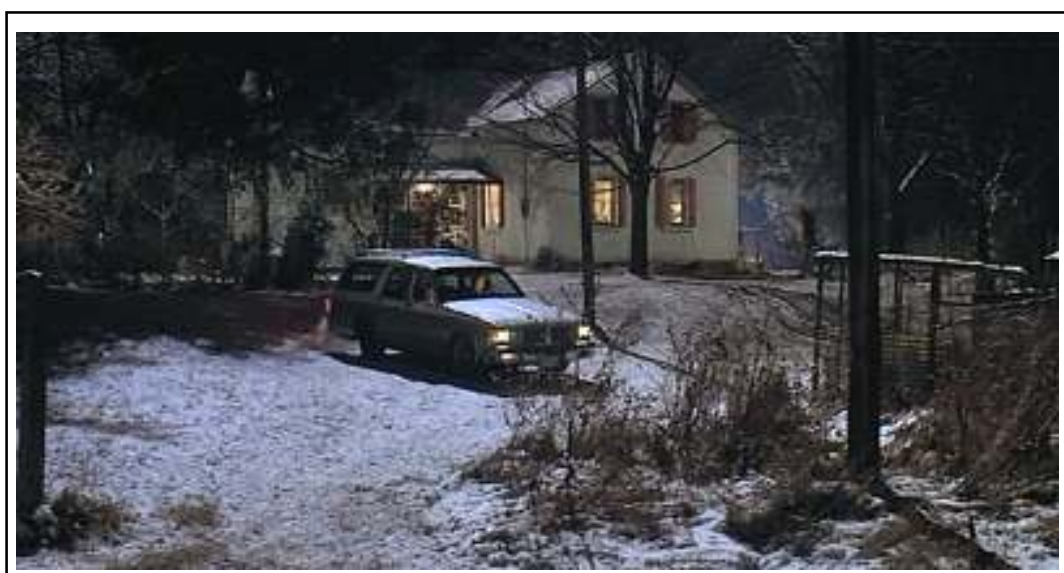

- Plan 1: La voiture se gare

- Son direct ( in) + musique extradiégétique (thème Joueur de

[ « The Sweet Hereafter », production : Alliance Communications Corporation / Ego Film Arts Tous droits réservés.]

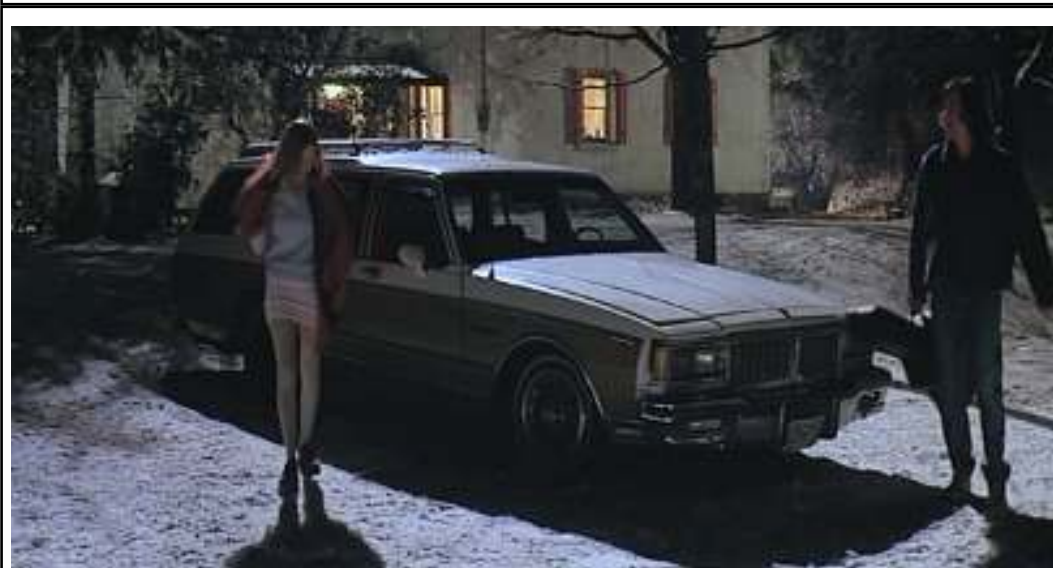
in) + musique extradiégétique + voix over de Nicole (lisant le conte)

[«The Sweet Hereafter », production : Alliance Communications Corporation / Ego Film Arts Tous droits réservés.]

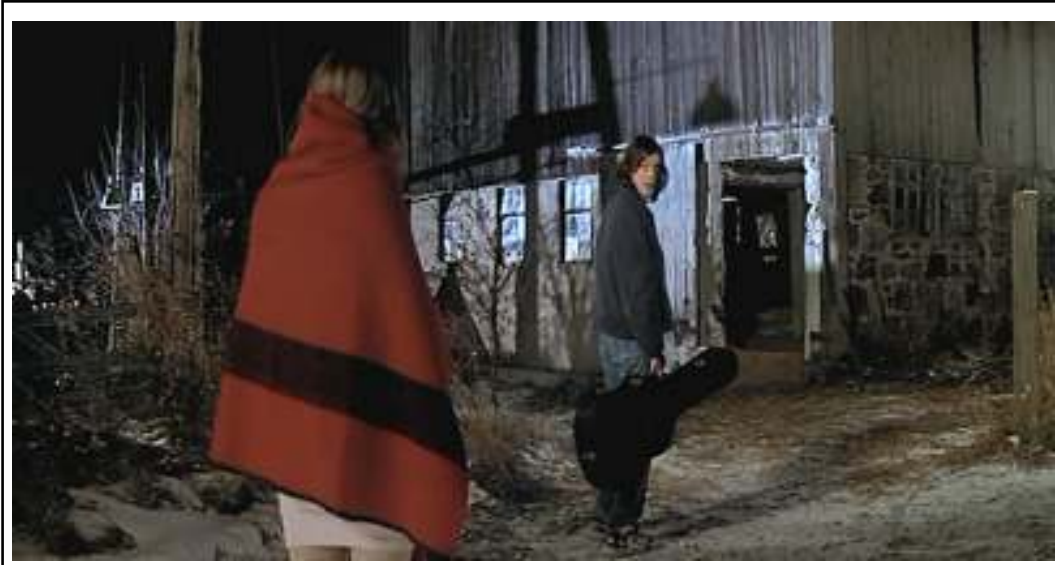

Nicole

s'arrête, Sam l'attend - musique extradiégétique + voix over de Nicole

["The Sweet Hereafter», production : Alliance Communications Corporation / Ego Film Arts Tous droits réservés.] 


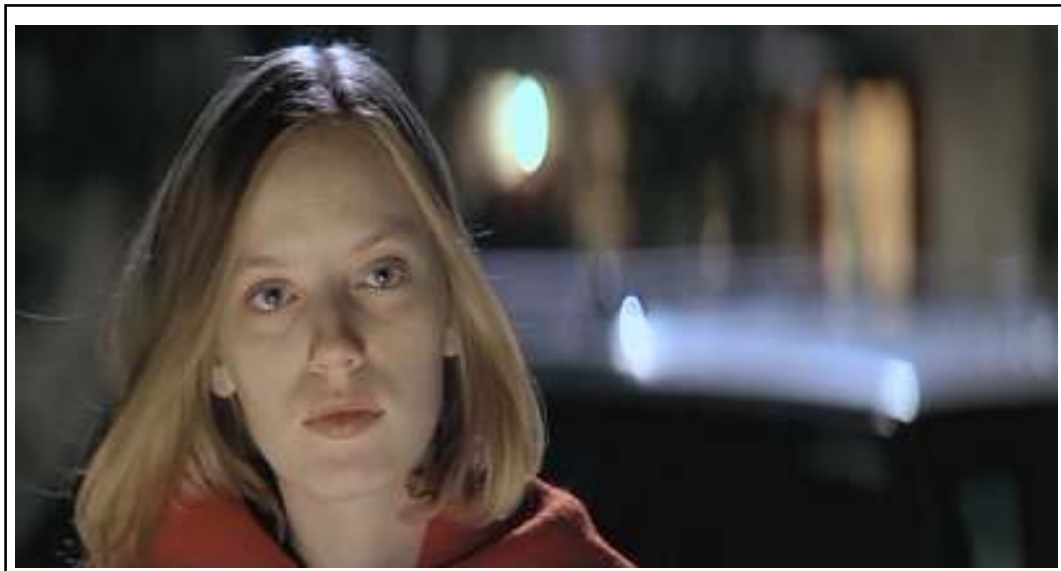

- Plan 4 (et plan 6) : Gros plan Nicole

- musique extra-

diégétique voix over de Nicole

[ « The Sweet Hereafter », production : Alliance Communications Corporation / Ego Film Arts . Tous droits réservés.]

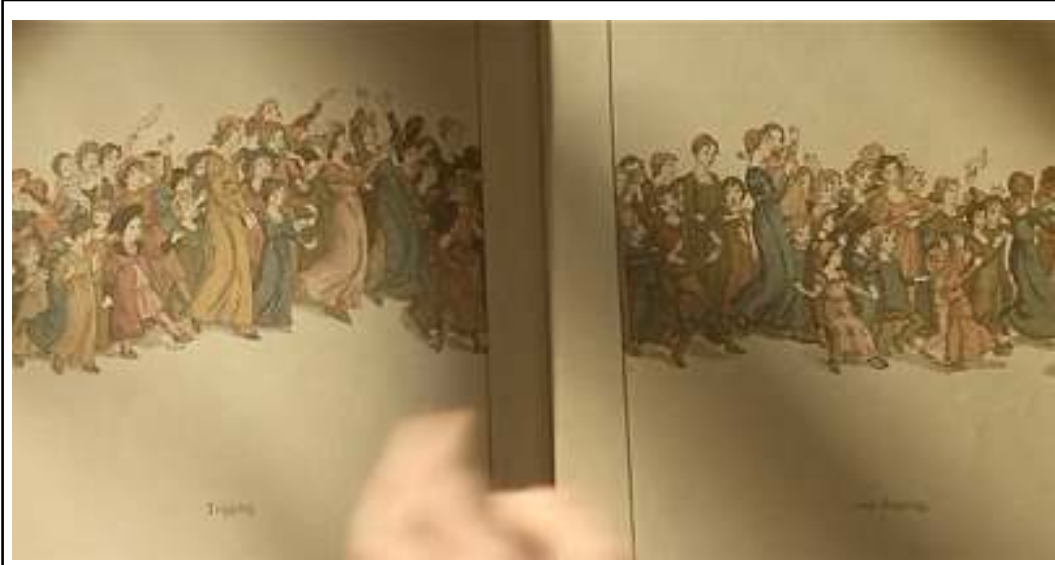

[«The Sweet Hereafter », production : Alliance Communications Corporation / Ego Film Arts Tous droits réservés.]

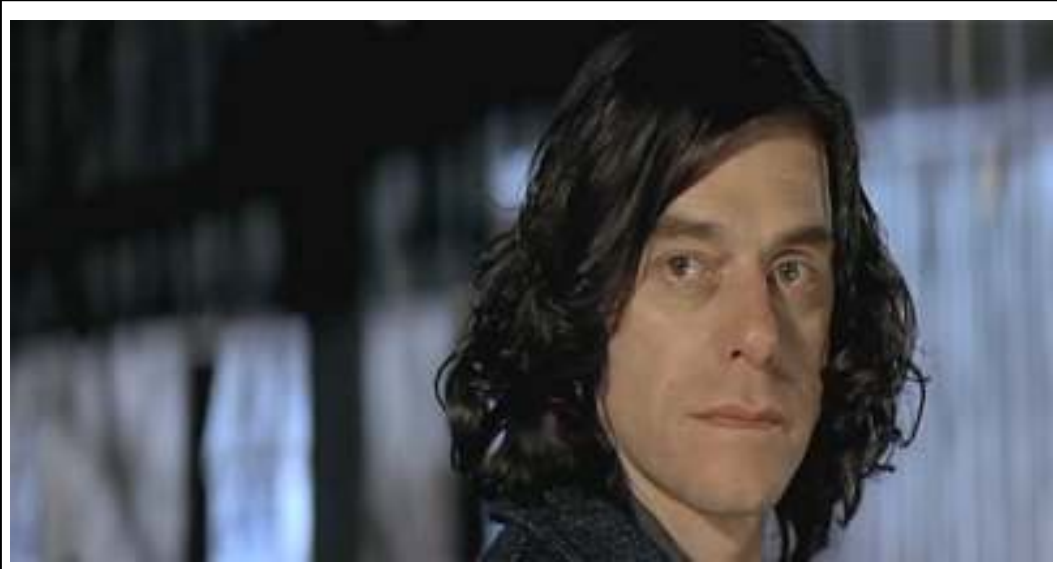
- Plan 5: Insert en gros plan du livre

- musique

extra-

diégétique + voix over de

Nicole

[«T The Sweet Hereafter », production : Alliance Communications Corporation / Ego Film Arts Tous droits réservés.] 


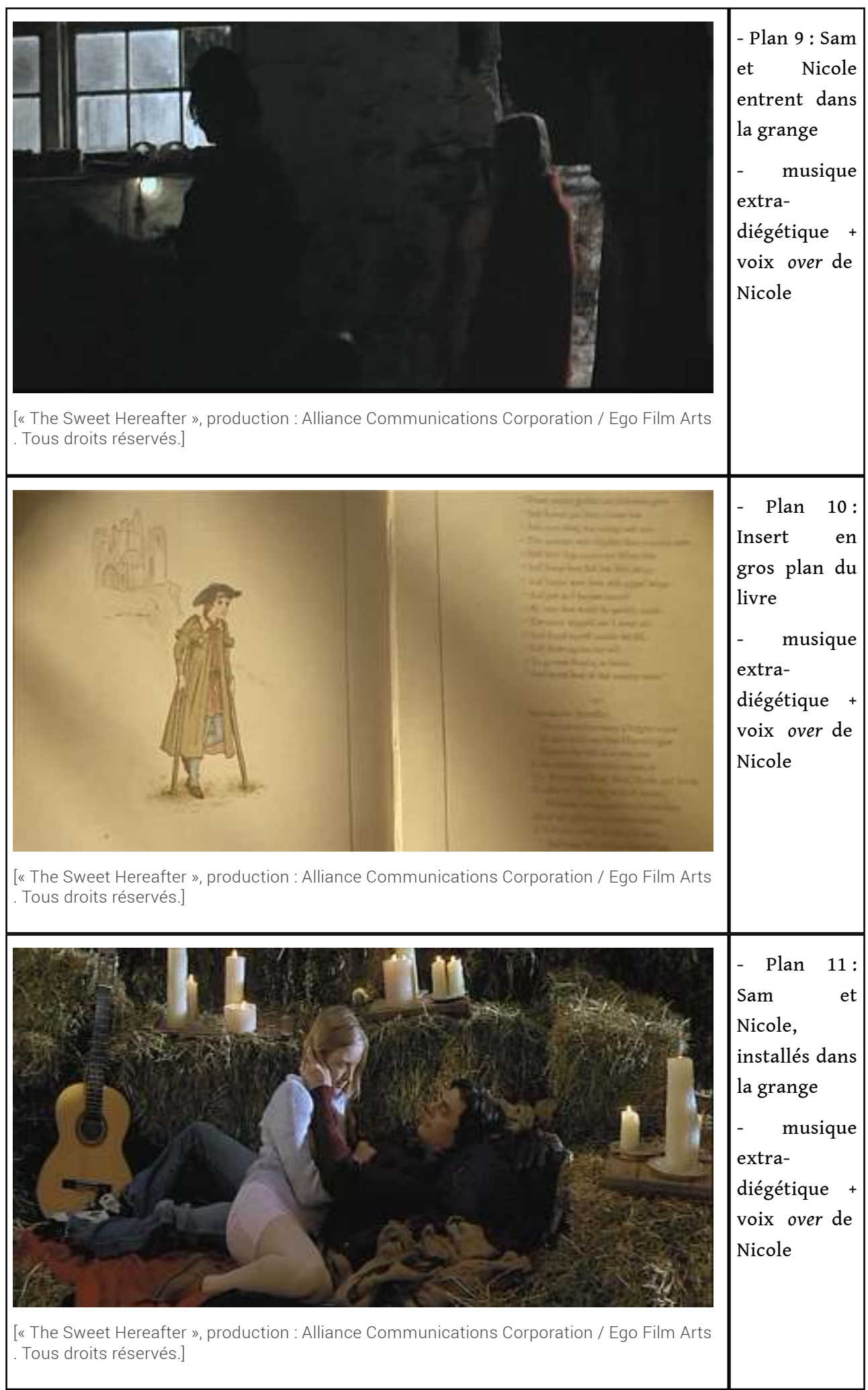

Cette séquence suit de près la temporalité de la scène précédente. Sam, le père de Nicole, vient la chercher chez Billy après le retour de ce dernier. Il la raccompagne à la maison, en passant auparavant par leur grange, lieu dans lequel il entretient une relation incestueuse avec elle. C'est le premier extrait du film dans lequel nous entendons la voix over de Nicole dans son sens premier : voix over parlée. 
39 Tandis qu'à l'image la voiture vient de se garer dans la pénombre, la porte côté conducteur s'ouvre, accompagnée d'un bruit. C'est à la suite de ce bruit que la voix over entame son récit avec une première correspondance audio-visuelle: There was a rusting that seemed like a bustling. Of merry crowds justling at pitching and husting ${ }^{9}$. Alors que Nicole sort de la voiture, le récit se poursuit avec une seconde correspondance audio-visuelle, le bruit de ses pas dans la neige : Small feet were pattering, wooden shoes clattering. Little hands clapping and little tongues chattering. And, like fowls in a farm-yard when barley is scattering ${ }^{10}$. À l'image, Nicole avance en direction de la caméra, lentement, puis s'arrête, ce qui nous montre ici un contre-emploi de l'écran sur le récit où les enfants courent: Out came the children running ${ }^{11}$. Le plan suivant montre Sam, la guitare de Nicole à la main, devant l'entrée de la grange. Cette dernière ne le suivant pas, il s'arrête et la regarde fixement: All the little boys and girls, with rosy cheeks and flaxen curls; Nicole apparait en gros plan : and sparkling eyes and teeth like pearls ${ }^{12}$. Alors qu'ici le texte évoque le sourire des enfants, Nicole nous apparaît sans expression à l'image, sans sourire, comme figée. C'est un second contrepoint audio-visuel. Apparaissent ensuite les pages du livre en insert et en gros plan : la voix over énonce le texte, elle devient comme in quelques instants. Tripping and skipping, ran merrily after, the wonderful music with shouting and laughter ${ }^{13}$. À l'image, le visage de Nicole, puis celui de Sam, apparaissent successivement, en gros plan. Cette troisième correspondance audio-visuelle nous présente Nicole comme l'enfant suivant le Joueur de Flûte (ici le Joueur de guitare). Le lien avec le livre étant clairement rappelé sur les images précédentes, le spectateur bascule à ce moment-là dans le monde enchâssé du Joueur de flute. Les plans suivant montrent Sam qui entre le premier dans la grange et Nicole qui le suit. La voix over poursuit avec une quatrième correspondance précise sur l'image. Pourtant, le récit vient d'être manipulé, quelques strophes dans le conte du Joueur de flute n'ont pas été lues. Le spectateur-auditeur n'en perçoit rien. La voix over dévoile un nouveau pouvoir : elle peut éluder une partie du récit. When, lo, as they reached the moutain-side, a wondrous portal opened wide, as if a cavern was suddently hollowed ${ }^{14}$.À cet instant, Sam pénètre dans la grange, il est immédiatement associé au Joueur de flûte. And when the Piper advanced and the children followed, and when all were in to the very last, the door in the mountain side shut fast ${ }^{15}$. Un gros plan du livre arrive en insert et nous montre l'enfant boiteux resté seul au village. Did I say, all? No: One was lame, and could not dance the whole of the way ${ }^{16}$ Nicole peut alors être associée à cet enfant. La voix over semble une nouvelle fois anticiper sur la suite du récit. Un peu plus loin, Sam est de nouveau associé au Joueur de flûte, c'est la cinquième correspondance audio-visuelle : I can't forget that I'm bereft of all the pleasant sights they see, which the Piper also promised $m e^{17}$ Tandis que Sam embrasse sa fille dans la paille, au milieu d'un décor illuminé à la bougie, la guitare est filmée, intégrée au décor, sortie de son étui, trônant à la verticale dans le cadre. La «promesse » de Sam peut-être comprise comme étant celle d'une répétition musicale dans la grange qui aura tourné à l'inceste.

Dans cette séquence, la voix over de Nicole est énonciatrice. Non plus lectrice, Nicole devient conteuse. Sa voix est plus posée, plus lente, plus articulée, moins spontanée que lorsqu'elle lisait le conte aux jumeaux Ansel. Nicole peut être perçue, à plusieurs reprises, comme le narrateur autodiégétique puisqu'elle est associée aux enfants suivant le Joueur autant qu'à celui qui n'accède pas au monde merveilleux promis par ce dernier. La corrélation entre la voix over et la diégèse implique Nicole et Sam dans les deux mondes parallèles. Le double récit est clairement ouvert et amorcé. La musique de fosse entendue dans cette séquence en atteste, c'est le thème du Joueur de flûte qui est joué. La voix over 
possède ici une fonction de génie dans le sens où elle donne accès au monde d'Hamelin, soit celui du Joueur de flûte, autant qu'elle anticipe sur l'avenir en présentant Nicole comme la future rescapée de l'accident. Le destinataire de cette voix over est le spectateur, sans conteste. L'image associe la diégèse à la métadiégèse, nous sommes dans un double récit où le spectateur-auditeur est amené par le narrateur embrayeur du monde enchâssé : Nicole. Le Joueur est matérialisé à l'écran, désigné par la voix over, il est figuré par Sam.

Extrait 5 (de $1: 29: 52$ à $1: 31: 51$, après l'accident de bus)

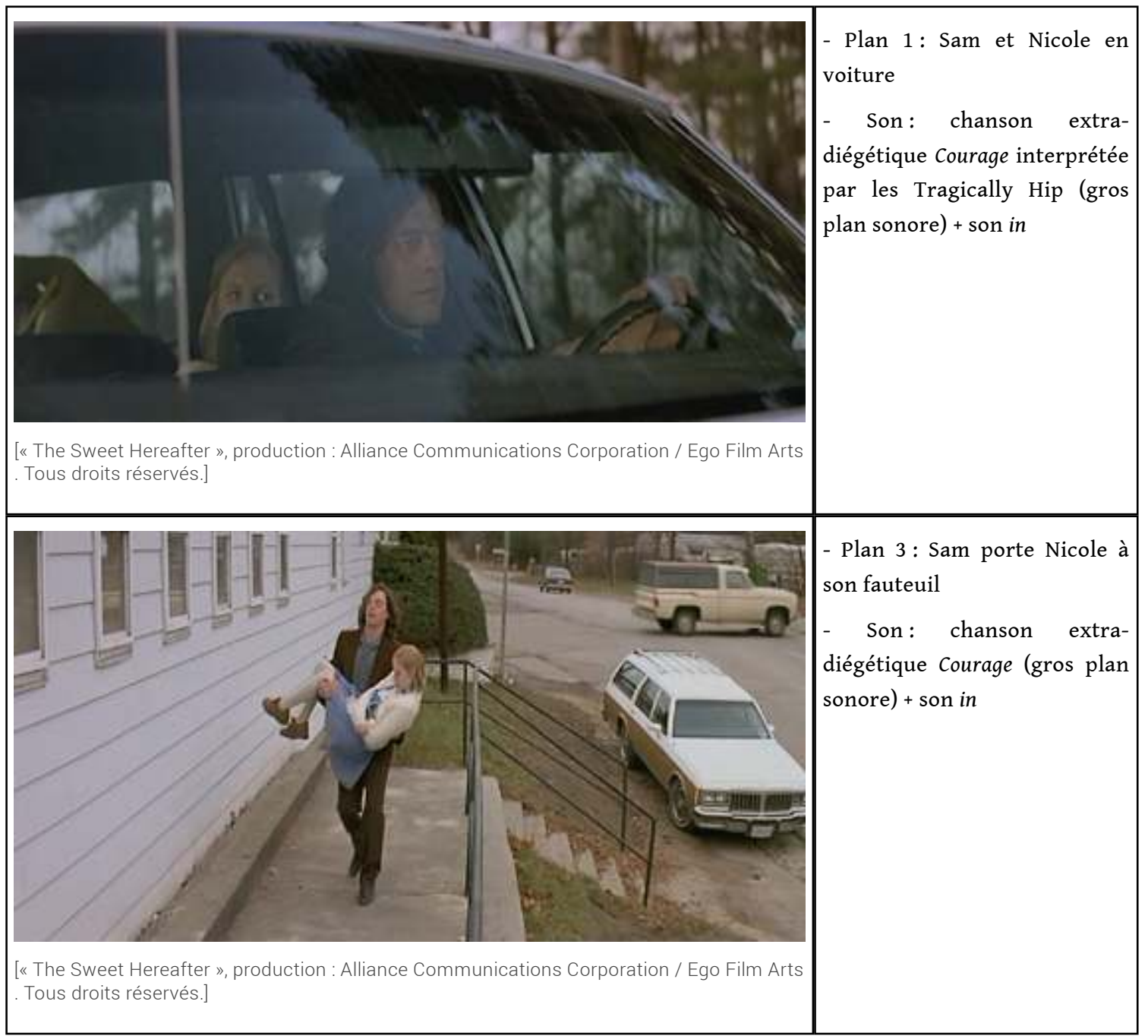




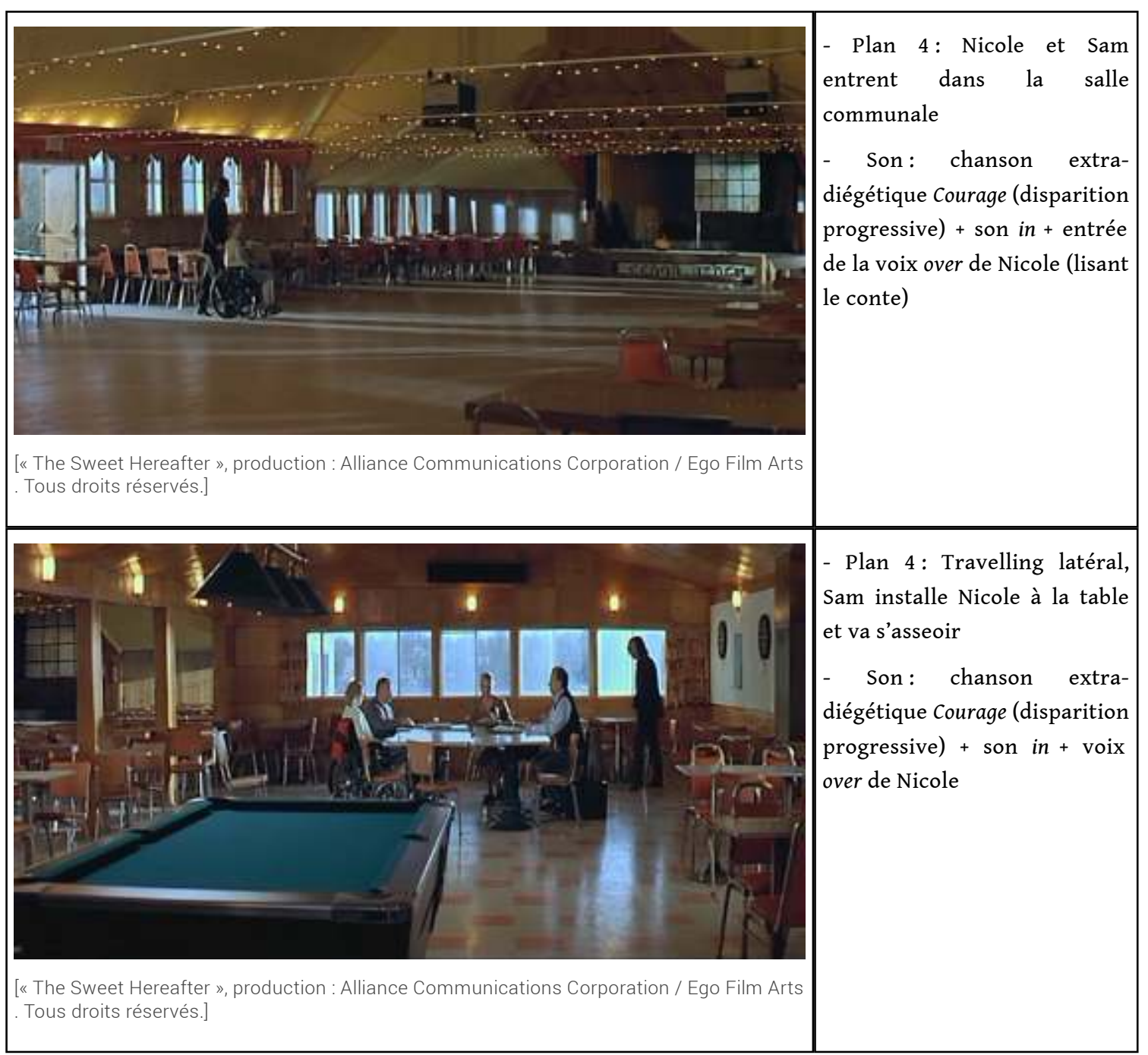

41 Nous sommes après l'accident, au jour de la déposition de Nicole citée comme principale témoin. Elle arrive en voiture avec son père vers la salle communale. En gros plan sonore, le spectateur-auditeur entend, pour la seconde fois la chanson Courage, diffusée dans la version originale des Tragically Hip. Elle a commencé sur la scène précédente, la veille de la déposition, en fond sonore (musique in) sous un dialogue entre Sam et Nicole où ce dernier cherchait à s'assurer qu'elle avait bien compris les enjeux financiers de ce procès. La musique, sur un crescendo, s'est poursuivie sur cette nouvelle scène où elle se révèle être extra-diégétique. La chanson devient le leitmotiv de Nicole qui a choisi de l'écouter la veille au soir, dans son lit: les paroles la poussent à la révolte. De nouveau, malgré la valeur narrative de cette chanson, aucun sous-titrage ne l'accompagne, privant le spectateur-auditeur non anglophone de l'accès à cette double narration.

La transition entre les deux séquences s'accompagne d'un crescendo sur cette première phrase : So there's no simple explanation for anything important any of us $\mathrm{do}^{18}$. Nicole et Sam apparaissent en voiture. And yea the human tragedy consists in the necessity of living with the consequences under pressure, under pressure ${ }^{19}$. Sam sort Nicole de la voiture, il la porte à bras le corps pour l'installer dans son fauteuil puisqu'elle a perdu l'usage de ses jambes dans l'accident. Courage, my word, it didn't come, it doesn't matter. Courage, your word, it didn't come, it doesn't matter. Courage, my word, it didn't come, it doesn't matter. Courage, it couldn't come at a 
worse time. It couldn't come at a worse time. It couldn't come at a worse time. Courage $e^{20}$ Progressivement, le volume de la chanson diminue tandis que Nicole entre dans la salle communale, conduite par son père.

Que nous apporte cette chanson ayant une valeur de voix over? Une fois de plus, elle anticipe sur l'action qui va suivre: la déposition. Le fait qu'elle soit diffusée dans sa version originale renforce ce ressenti. En écoutant non plus ses reprises mais la version rock des Tragically Hip, Nicole s'imprègne de la violence des paroles et de la mélodie. Le chanteur s'adresse avant tout à Nicole lorsqu'elle écoute sa chaîne hi-fi (musique in) dans son lit, la veille au soir. Elle est ensuite diffusée (musique over) pour le spectateurauditeur, livrant des clefs de lecture sur le double récit qui s'installe. Nicole est sous pression parce que sa décision est prise : elle va faire une fausse déposition. La première phrase peut être vue comme une justification de l'acte que Nicole s'apprête à faire. Cette chanson la prépare à devenir le joueur de flûte. La seconde phrase explique la punition que Sam va recevoir de Nicole : quoi qu'il fasse maintenant, il n'effacera jamais la blessure qu'il a causée à sa fille. Enfin, le refrain s'adresse à Nicole. Le chanteur la conseille : «Courage, c'est ton mot », il l'incite à aller au bout de ce qu'elle a décidé.

Tandis que Nicole et Sam pénètrent dans le hall de la salle, la voix over de cette dernière retentit.

Dans le scénario, une voix over était déjà présente : Nicole évoquait la relation incestueuse avec son père et sa révolte personnelle. À la lecture audio-visuelle de la scène filmée, nous comprenons que ce passage initial a été supprimé au profit de la version originale de Courage qui, comme nous venons de le voir, retranscrit très bien ce même discours. La voix over parlée et donc remplacée par une voix over chantée qui n'est plus celle de Nicole. Alors qu'elle rejoint la table de la déposition, la voix over de Nicole reprend la lecture du conte du Joueur de flûte: When, lo, as they reached the moutain-side, a wondrous portal opened wide, as if a cavern was suddently hollowed. And when the Piper advanced and the children followed, and the when all were in to the very last, the door in the mountain side shut fast. Did I say, all? No: One was lame, and could not dance the whole of the way ${ }^{21}$. On reconnaît la première intervention de Nicole en voix over, dans la grange. L'écho avec cette scène précédente, s'il nous présente le même discours, introduit une nouvelle lecture de l'image. Nicole est de nouveau le narrateur énonciateur, la conteuse en position autodiégétique. Le double récit nous indique que ce n'est plus Sam le Joueur de flûte, mais bien Nicole. Sam devient l'enfant boiteux qui n'entre pas dans le monde enchanté du Joueur. À l'image, Nicole, en fauteuil roulant devance son père qui, inévitablement, la suit; il est ensuite le dernier à s'asseoir, après avoir installé sa fille. Par ailleurs, au moment de leur entrée dans la salle communale, la caméra laisse apercevoir une estrade dans le fond du cadre. Alors que Nicole passe devant, on s'imagine qu'elle a déjà pu chanter ici. Ce lieu semble donc être le repère de Nicole et non plus celui de Sam. Enfin, sur la scène, une guitare sortie de son étui est disposée à la quasi verticale, rappelant son instrument de prédilection. Nous sommes dans l'univers de la musicienne, soit de la Joueuse. Là encore, la voix over matérialise à l'écran les personnages énoncés, le Joueur est figuré par Nicole, l'exclu est Sam. Ce discours s'adresse au spectateur afin de lui permettre d'accéder à ce double récit avec le monde du Joueur de flûte.

Extrait 6 (de $1: 34: 07$ à $1: 37: 30$, après l'accident de bus)

Avant le mensonge de Nicole 


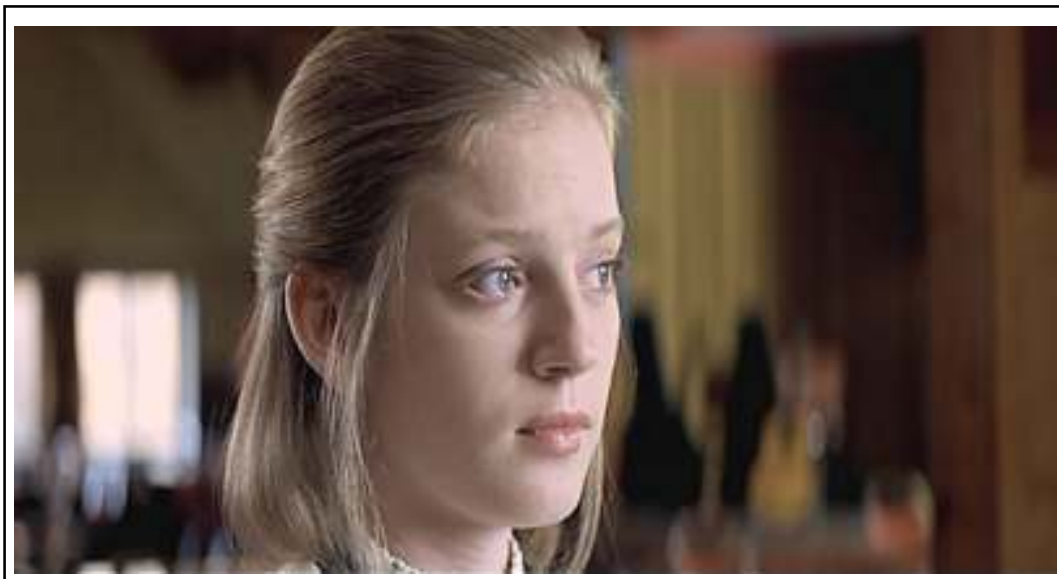

- Gros plan de Nicole qui fixe Sam

- Son in + voix over de Nicole (lisant le conte)

[ « The Sweet Hereafter », production : Alliance Communications Corporation / Ego Film Arts Tous droits réservés.]

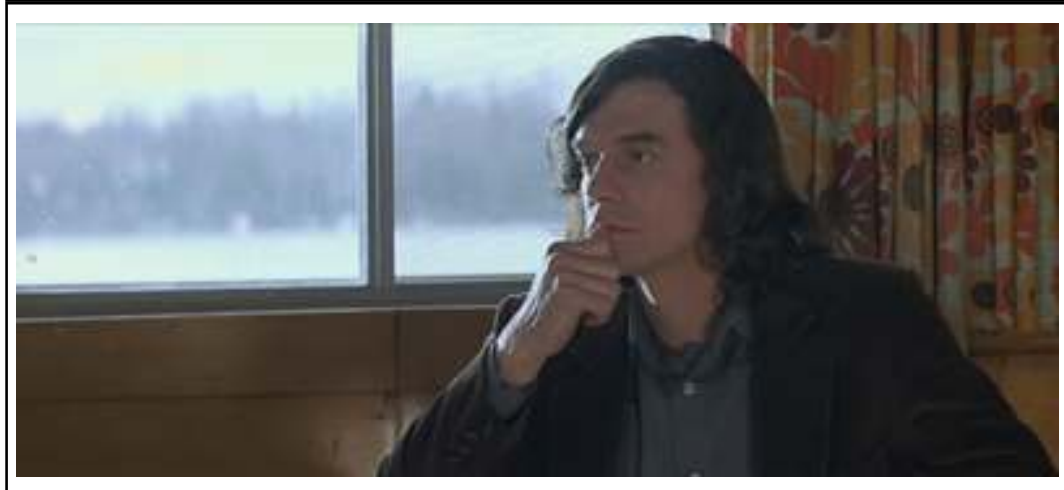

[« The Sweet Hereafter », production : Alliance Communications Corporation / Ego Film Arts Tous droits réservés.]

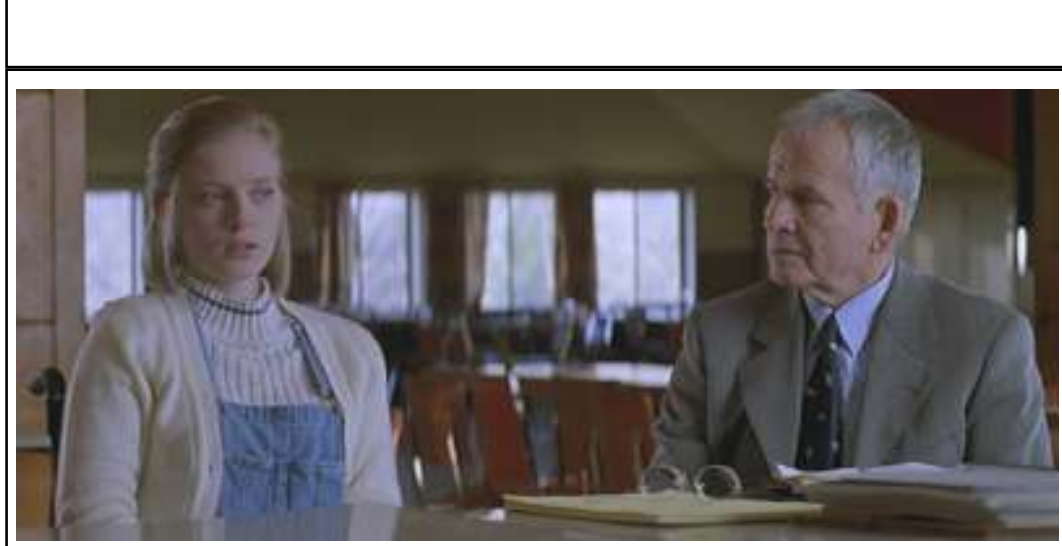
latéral vers Sam

- Son in + voix over de Nicole (lisant conte) musique extradiégétique (thème Joueur flûte) Nicole, à côté Mitchell, ment - Son in + musique extradiégétique

["The Sweet Hereafter», production : Alliance Communications Corporation / Ego Film Arts Tous droits réservés.] 


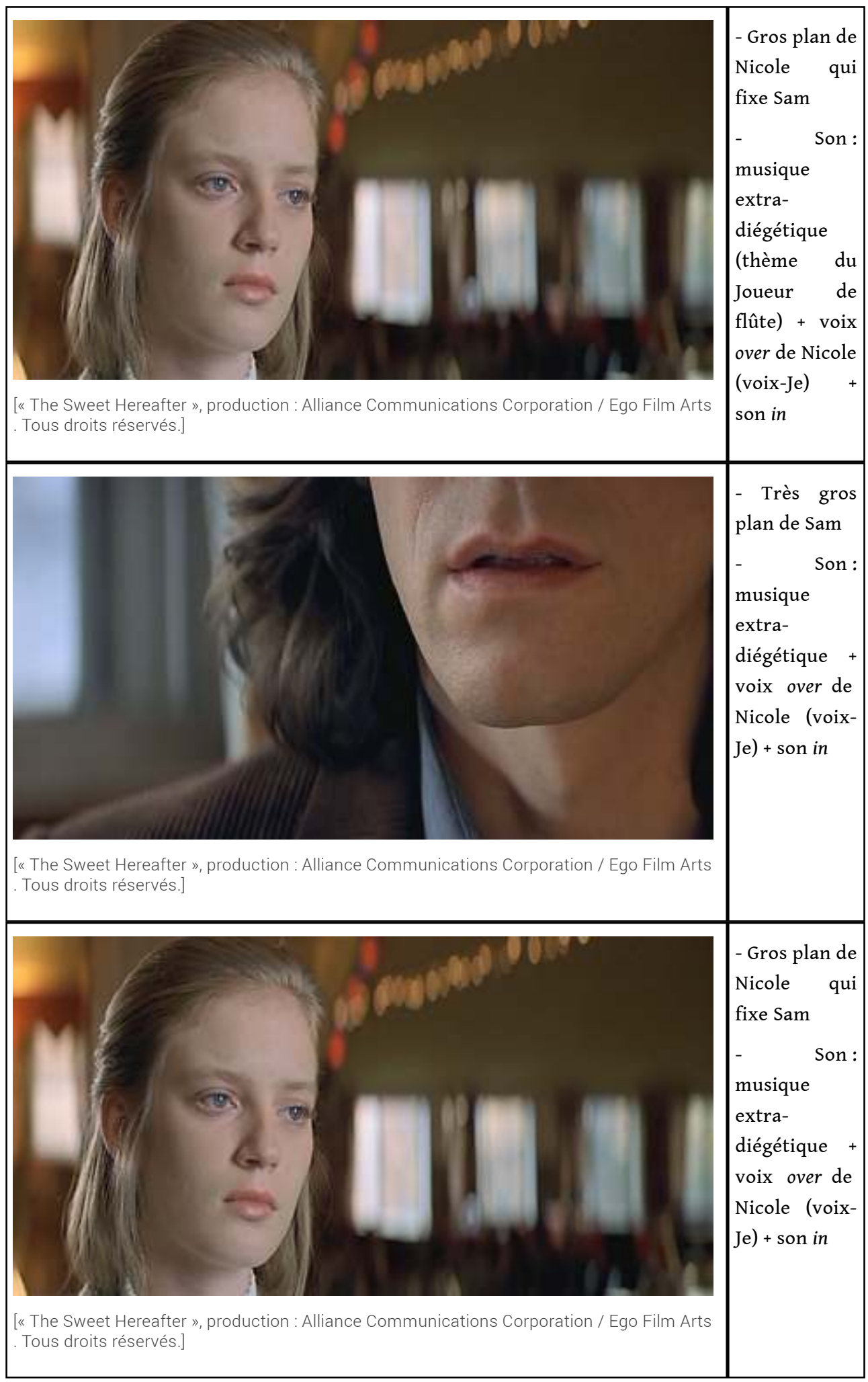

La scène se poursuit. C'est l'heure de la déposition. L'avocat, Mitchell Stephens est aux côtés de Nicole. Son père, en retrait, au fond de la salle lui fait quasiment face. Pendant que Nicole le regarde fixement, la voix over poursuit la lecture du conte. It's dull in our town since my playmates left! I can't forget that I'm bereft of all the pleasant sights they see, which the Piper also promised $m e^{22}$. Alors que Nicole et Sam viennent d'échanger des regards sur 
cette voix over, on peut se remémorer qu'elles ont déjà été prononcées dans la grange. Ces paroles reviennent comme un refrain. Elles renvoient à la scène où Sam embrassait Nicole. Cette voix over renvoie également au monde du Joueur de flûte. Nicole tient ici sa revanche. C'est alors qu'elle fait une fausse déclaration. Son mensonge ne permet pas d'engager le procès attendu par Mitchell et Sam. Tout au long de sa déclaration, elle regarde Sam fixement, assumant frontalement cette riposte. En arrière-plan, la guitare acoustique apparaît floutée. Nicole entre dans la figure du Joueur de flûte afin de sauver son village d'un procès destructeur. Elle se venge également de celui qui a joué dans sa vie le rôle dévastateur du Joueur. Alors que Sam, ébahi, reste littéralement bouche bée, le cadre change autour de Nicole, filmée en gros plan. La guitare a disparu de l'image. Le thème musical du Joueur de flûte retentit de façon extra-diégétique pour la dernière fois dans le film. La voix over de Nicole poursuit: And why I lied? He only knew. But from my lie, the stay come true. Those lips from which he does this tune were frozen as the winter moon ${ }^{23}$. Si ces phrases sont énoncées en ver, à la façon du conte, elles n'appartiennent en réalité pas au Joueur de flute de Hamelin. Nicole sollicite ici ses propres mots à travers la voix over. Le commentaire collant précisément à Sam semble représenter le monologue intérieur de Nicole en voix-Je.

Pour la première fois, Nicole est locutrice et non plus énonciatrice. C'est son propre discours que l'on entend. Elle est, de nouveau le narrateur autodiégétique : elle est, à travers son mensonge, non plus la figuration, mais l'incarnation du Joueur. La voix over ouvre ainsi les portes au spectateur-auditeur d'un nouveau monde délivré du Joueur de flûte et du procès : le monde des Beaux Lendemains. Le discours s'adresse précisément au spectateur-auditeur. Dans cette scène, la voix over initialement prévue au scénario décrivait la déception du père face à cette perte économique. Nicole, en écrivant son propre discours, pour la première fois dans le récit, change son cours.

Extrait 7 (de $1: 40: 26$ au générique fin, après l'accident de bus)

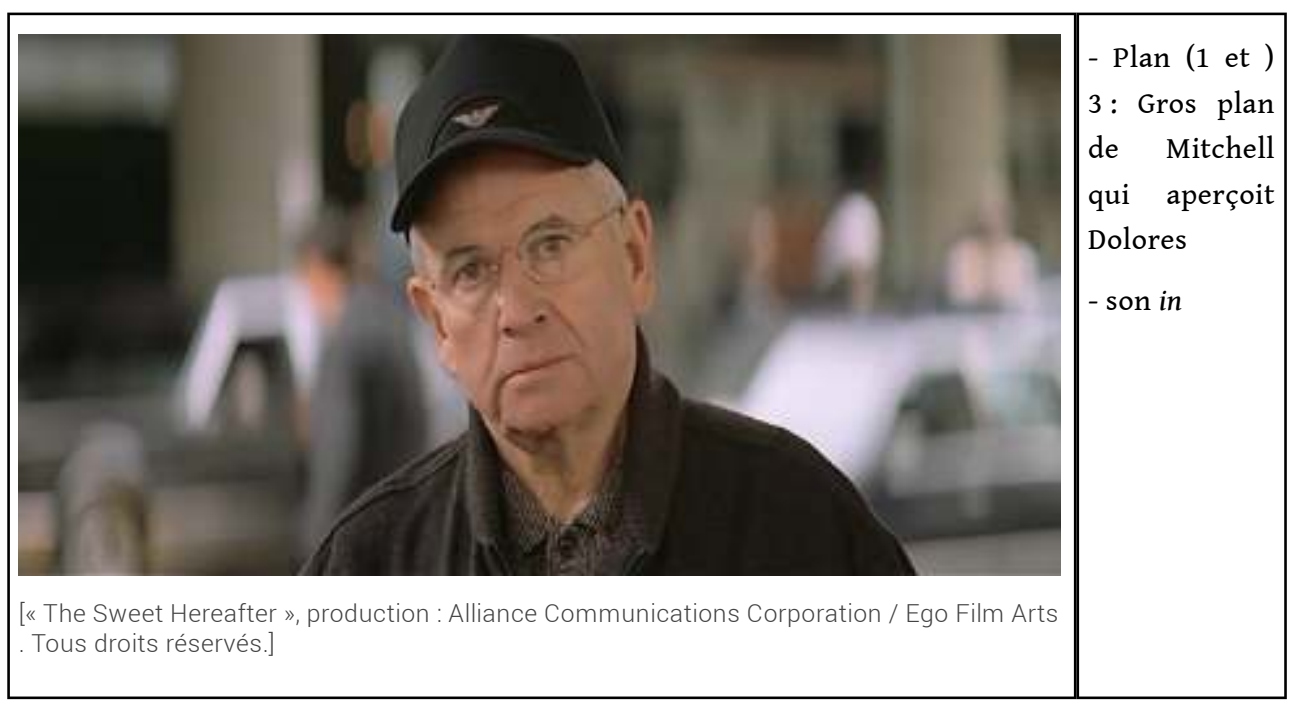




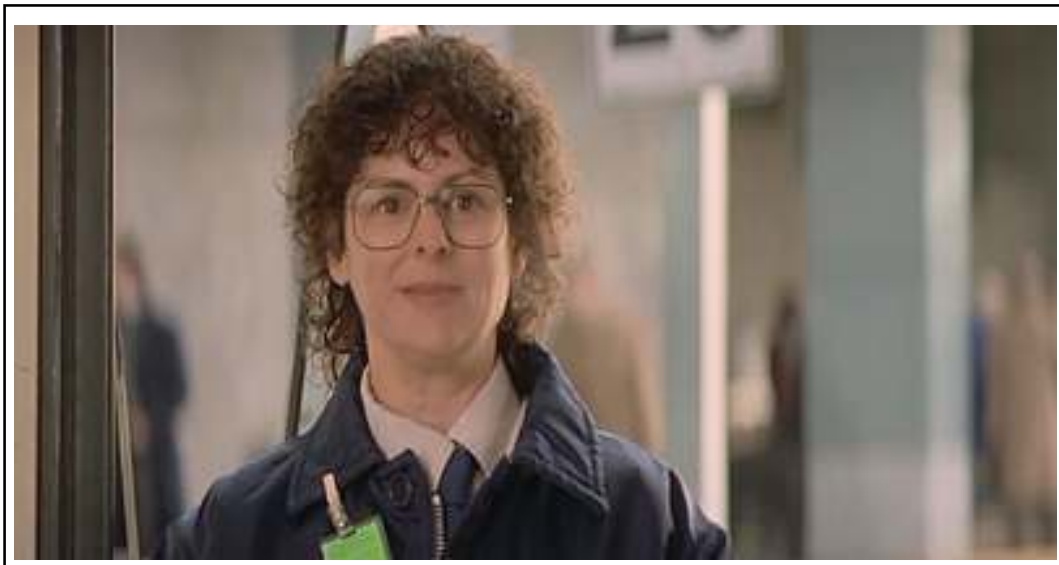

- Plan (2 et) 4: Gros plan de Dolores qui aperçoit Mitchell - son in + voix over (voix-Je) de Nicole

[ The Sweet Hereafter », production : Alliance Communications Corporation / Ego Film Arts Tous droits réservés.]

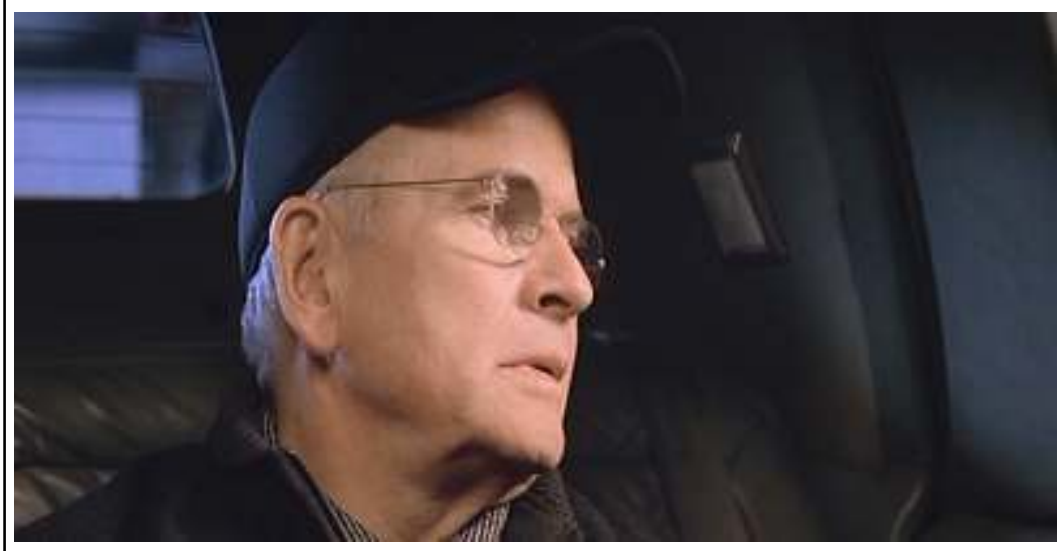

- Plan 5 : Gros plan de Mitchell dans le taxi voix over (voix-Je) de Nicole musique extradiégétique (thème des [« The Sweet Hereafter », production : Alliance Communications Corporation / Ego Film Arts Tous droits réservés.] Beaux

Tous droits reserves.] Lendemains)

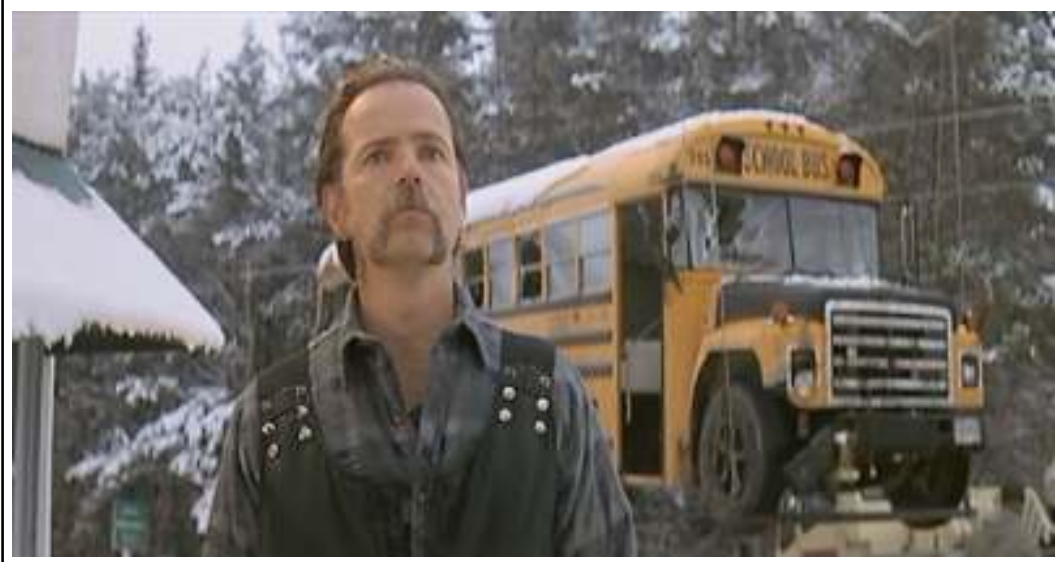

- Plan 6 : Billy devant l'enlèvement du bus accidenté - voix over (voix-Je) de Nicole musique extradiégétique

[«The Sweet Hereafter », production : Alliance Communications Corporation / Ego Film Arts Tous droits réservés.] 


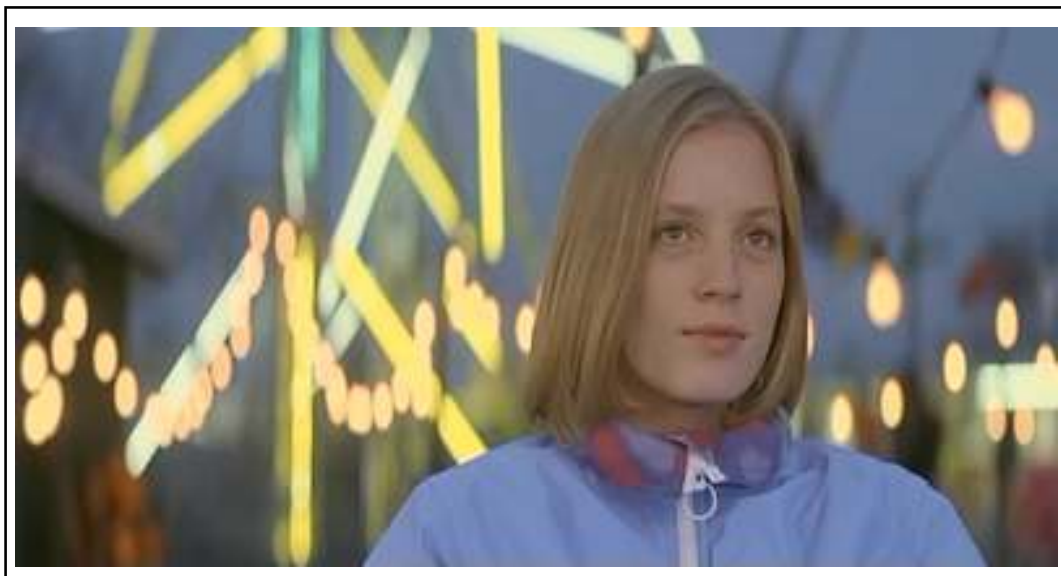

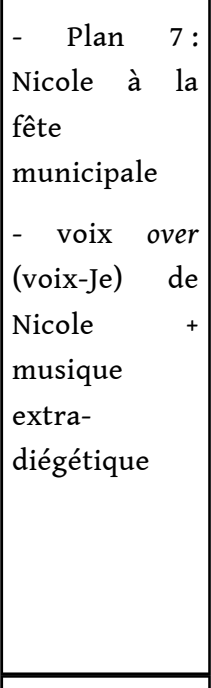

"The Sweet Hereafter », production : Alliance Communications Corporation / Ego Film Arts Tous droits réservés.]

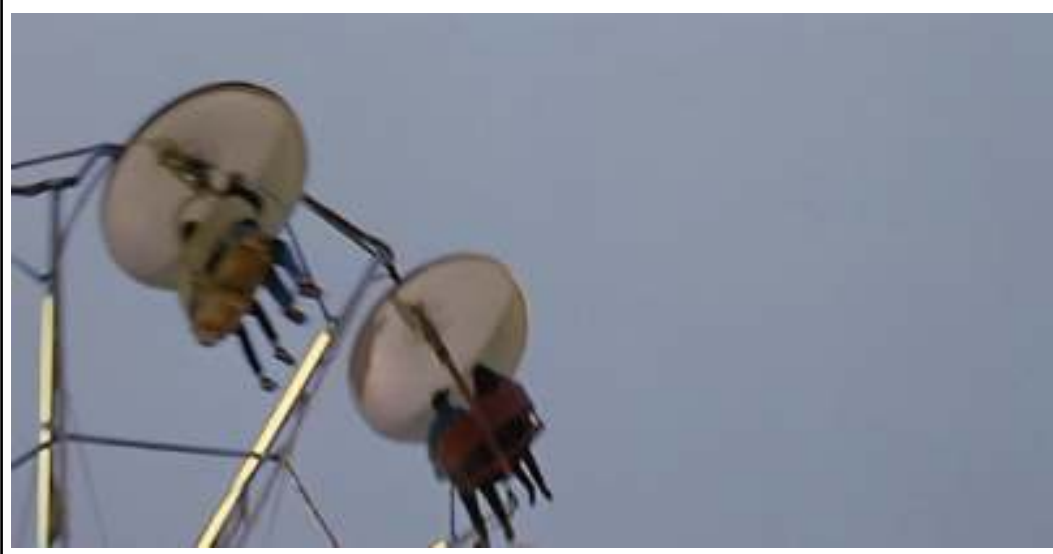

- Plan 8: Vue du ciel et d'un manège en action

voix over (voix-Je) de Nicole musique extradiégétique

[ The Sweet Hereafter », production : Alliance Communications Corporation / Ego Film Arts Tous droits réservés.]

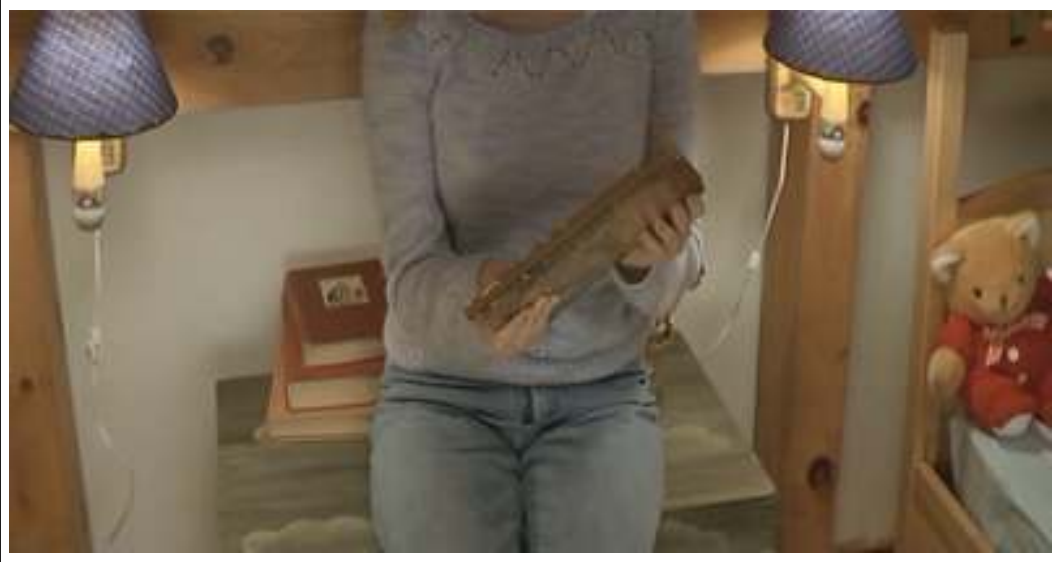
des jumeaux Ansel, referme le conte Joueur flûte extra-

[«T The Sweet Hereafter », production : Alliance Communications Corporation / Ego Film Arts Tous droits réservés.]

diégétique (thème des Beaux Lendemains)

La dernière séquence du film nous promène dans la diégèse une dernière fois. Mitchell Stephens, au sortir de l'aéroport, aperçoit Dolorès qui a quitté Sam Dent pour reprendre son travail de chauffeur de bus en ville. C'est alors que la dernière intervention en voix over de Nicole se fait entendre: As you see her, two years later, I wonder if you realize 
something. I wonder if you understand that all of us - Dolores, me, the children who survived ${ }^{24} \grave{\mathrm{A}}$ l'image, apparait Billy Ansel face au bus accidenté qui part à la casse. The children who didn't - that we're all citizens of a different town now. A place with his own special rules, and his own special laws. A town of people living in the sweet hereafter ${ }^{25}$. Une fois de plus, ces phrases sont la propre composition de Nicole. Parce qu'elle interpelle directement le spectateurauditeur, c'est une voix over qui se rapproche de l'aparté sonore. En effet, Nicole n'est pas directement filmée en train de nous parler (Châteauvert, 1996 : p. 141). Elle est locutrice, loin de l'évocation du conte, elle décrit le nouvel univers dans lequel les habitants de Sam Dent vivent désormais grâce à elle. Elle nous donne accès au monde des Beaux Lendemains. Le plan suivant nous amène à la fête municipale, celle-là même pour laquelle Nicole répétait au début du film. La voix over poursuit: Where waters gushed and fruit-tree grew, and flowers put forth a fairer hue, and everything was strange and new.Everything was strange and $n e w^{26}$. Cette dernière intervention vocale énonce un passage du conte déjà récité dans la scène de la grange (fin de l'extrait 4). Cela fait référence au lieu que le Joueur de flûte avait promis et confirme donc que le village est désormais délivré.

Nicole redevient l'énonciatrice, la lectrice du conte. Les dernières images du film sont la suite de l'extrait 3, jamais diffusé auparavant : Nicole est dans la chambre de Jessica et Mason. Elle vient de terminer la lecture du conte (comme nous venons de l'entendre, et bien que ce ne soit pas réellement la dernière phrase écrite). Elle referme alors le livre et le repose sur la table de chevet. Les jumeaux dorment paisiblement. Ce retour dans la diégèse, à la veille de l'accident, permet au récit enchâssé de se conclure avec la fermeture du livre. Les guillemets de la voix over lisant le conte peuvent alors se refermer (Chion, 2003 : p. 76) et le Joueur disparaître de Sam Dent. Nicole apparaît une dernière fois comme la libératrice puisque c'est elle qui ferme l'accès au double récit installé.

Dans le film De Beaux Lendemains, la narration est principalement menée par la bande sonore : à travers la musique (thème de la communauté de Sam Dent, thème du Joueur de flûte, thème des Beaux Lendemains, reprises des chansons pop rock canadiennes de l'époque) ; à travers la chanson (Courage des Tragically Hip) et essentiellement à travers la voix over.

51 La déconstruction du récit et les allers-retours dans les différentes temporalités de la diégèse tendent à attirer l'attention du spectateur-auditeur sur la dimension sonore et vocale du film, élément fondateur de la narration. C'est bien le caractère non linéaire du récit filmique qui permet au spectateur, perdu dans le récit, d'accepter plus facilement la transition vers les différents mondes enchâssés.

Nous avons isolé, dans le film, un procédé de voix over chantée : il est apparu dans ces extraits qu'elle tient les mêmes fonctions que la voix over parlée en anticipant principalement sur l'action. Elle conserve, pour autant, l'une des fonctions principales des chansons dans les films: la force de proposition qu'elle insuffle à qui l'écoute (en l'occurrence, Nicole). Courage est une musique influente. C'est une voix over chantée opérante pour Nicole, d'où la nécessité de bien comprendre les paroles, malheureusement non traduites dans la version originale sous-titrée.

Nous avons également étudié les fonctions et les déclinaisons de la voix over parlée de Nicole: sa voix over lue nous donne accès au monde du Joueur de flûte, tandis que sa voix over parlée nous ouvre l'accès au monde des Beaux Lendemains (la ville de Sam Dent libérée du procès). Pour reprendre la théorie de Jean Châteauvert, Nicole, à travers sa 
voix over, est bien le sujet embrayeur qui nous permet d'amorcer les mondes enchâssés du récit. populaires de son pays: le Canada. Elle lit également le conte de Joueur de flute. Le spectateur-auditeur trouve dans la diégèse à se connecter à son monde réel par le biais de Nicole. Cette dernière devient alors un personnage dont il peut aisément valider les propos et agissements. Elle apparait comme le meilleur guide du film pour suivre le récit. Elle est notre référent dans la diégèse.

57 - le monde de Hamelin: c'est sans doute notre confiance en Nicole qui nous permet d'accepter aussi facilement le glissement dans ce nouveau monde métadiégétique (Genette : 1972). Cette dernière y évolue sans difficulté par son statut de musicienne et celui de conteuse.

58 - le monde des Beaux Lendemains : il coexiste avec celui de Sam Dent, c'est la vie après l'accident, Sam Dent libérée du procès ou encore, un nouveau monde diégétique. Nous y avons accès par la voix-Je de Nicole qui a su réécrire l'avenir de son village.

59 Pour conclure, Nicole nous balade à travers ces différents mondes grâce à sa voix over tout en sollicitant des référentiels présents dans notre propre monde (les chansons, le conte du Joueur de flûte...). Elle nous fait ainsi glisser dans le double récit installé entre la diégèse et la métadiégèse. Sa voix over s'inscrit elle aussi, comme la chanson, dans un principe de circulation. C'est bien elle qui nous entraîne dans les différents mondes.

60 Précisons, pour finir, que le générique de fin donne à entendre, en intégralité, la version reprise et chantée de Courage par Nicole. Si elle est présentée comme une chanteuse dès le début du film, le réalisateur la sollicite une fois de plus dans ce rôle à la fin du récit. Elle dévoile au spectateur-auditeur son leitmotiv dont les paroles offrent les dernières explications possibles sur son mensonge.

61 Atom Egoyan, en supprimant la majorité des interventions conventionnelles en voix over dans son film, explore de nouvelles sources de narration. Nous avons pu vérifier que la voix over est sollicitée selon trois différentes propositions sonores, traversant la frontière des catégories parlée à chantée, puis déclinée de la voix lue à parlée. Cette dernière est sollicitée de façon minoritaire dans le film.

62 Nous pouvons ainsi concevoir que la voix over est appelée à se développer dans les récits filmiques sous des formes toujours plus innovantes que la voix over parlée. La voix over silence sollicitée en plein milieu de sa phrase, ou encore la voix over bruit, dont un bel exemple a été montré par Alain Boillat - avec le sonovox dans A Letter to three Wive -sont caractéristiques des deux dernières catégories sonores non exploitées dans De Beaux Lendemains. Ouvrir les frontières sonores de la voix over permettrait au cinéma contemporain de développer un nouveau champ d'exploration comme une valeur ajoutée à la mise en scène cinématographique. 


\section{NOTES}

1. Titre original du film: The Sweet Hereafter

2. Didier Péron, «Des lendemains qui hantent. De beaux lendemains d'Atom Egoyan, funèbre et superbe ", Libération, 08/10/1997.

3. Russell Banks, De beaux lendemains, traduit de l'américain par Christine Le Bœuf, Arles, éd. Actes Sud, 1997.

4. Robert Browning, The pied piper of Hamelin (Le Joueur de flûte de Hamelin), llustrated by Kate Greenaway, London Frederick Warne ans Co., and New York, 1888.

5. «Entretien avec Atom Egoyan » par Michel Ciment et Philippe Rouyer, Positif, oct. 1997, n 440, p. 17

6. Traduction proposée : Parle un peu plus doucement, travaille en faisant un peu plus de bruit, fais feu moins souvent mais avec plus de soin et chante un peu plus doucement et aime un peu plus longtemps et bientôt tu y arriveras. (Refrain) Ici tout ce qu'on a, c'est le ciel. Tout ce que le ciel est, c'est bleu. Tout ce que le bleu est maintenant, c'est juste une couleur de plus.

7. Traduction proposée : Regarde la bande à travers un bouquet de danseurs.

8. Traduction proposée : Rapidement, suis l'inconnu avec quelque chose de plus familier. Rapidement, quelque chose de familier. Courage, mon mot, il n'est pas venu, ce n'est pas grave.

9. Traduction proposée : Aussitôt on entend un bruissement et résonnent des cris joyeux d'enfants. Il se fit grande agitation, puis vint la précipitation.

10. Traduction proposée: De petits pieds si fort pressés qu'ils faisaient leurs sabots claquer. Petites mains applaudissant, petites langues babillant comme font poussins en poulailler à l'heure de la becquée.

11. Traduction proposée : Les enfants de la ville entière ainsi donc se précipitèrent.

12. Traduction proposée: Petites filles aussi bien que petits garçons, aux joues roses et aux boucles blondes, au regard pétillant, aux dents éclatantes,

13. Traduction proposée : trébuchant, dansant, gambadant. Ils suivirent en s'amusant le Joueur aux airs fascinants.

14. Traduction proposée: Quand ils furent devant la pente, on vit s'ouvrir dans la paroi une porte géante comme une faille soudain béante.

15. Traduction proposée : Le Joueur s'y enfonça suivi par tout le petit monde. Et quand ils furent entrés jusqu'au dernier, la faille se referma.

16. Traduction proposée : Ai-je tout dit? Non. L'un deux, boitant bas, trop lent, n'entra pas.

17. Traduction proposée : Je ne peux oublier que je suis privé du pays enchanté où ils s'en sont allés, et qu'à moi aussi, le Joueur avait promis.

18. Traduction proposée : Alors il n'y a pas d'explication simple pour quoi que ce soit d'important que l'un de nous puisse faire.

19. Traduction proposée: Oui, la tragédie humaine consiste dans la nécessité de vivre avec les conséquences sous la pression, sous la pression.

20. Traduction proposée : Courage, c'est mon mot, ce n'est pas venu, ce n'est pas grave. Courage, c'est ton mot, ce n'est pas venu, ce n'est pas grave. Courage, mon mot, ce n'est pas venu, ce n'est pas grave. Courage, ça n'aurait pas pu venir à un pire moment. Ça n'aurait pas pu venir à un pire moment. Ça n'aurait pas pu venir à un pire moment. Courage.

21. Traduction proposée: Quand ils furent devant la pente, on vit s'ouvrir dans la paroi une porte géante comme une faille soudain béante. Le Joueur s'y enfonça suivi par tout le petit monde. Et quand ils furent entrés jusqu'au dernier, la faille se referma. Ai-je tout dit? Non. L'un deux, boitant bas, trop lent, n'entra pas. 
22. Traduction proposée: Quel ennui dans notre village depuis que mes amis sont partis! Je ne peux oublier que je suis privé du pays enchanté où ils s'en sont allés, et qu'à moi aussi, le Joueur avait promis.

23. Traduction proposée: Pourquoi ai-je menti? Lui seul le sait. Mais de mon mensonge sortit une vérité. Les lèvres dont il tirait cet air étaient plus froides que lune d'hiver.

24. Traduction proposée : En la revoyant, deux ans plus tard, je me demande si vous comprenez. Si vous comprenez que tous autant que nous sommes, Dolores, moi, les enfants qui ont survécu

25. Traduction proposée : ceux qui ont disparu, sommes désormais citoyens d'une autre contrée. Un pays avec ses propres règles et ses lois. Où les gens vivent de beaux lendemains.

26. Traduction proposée : Où les sources sont claires, où abondent vergers et rivières, où les fleurs ont couleur plus belle, où toute chose est nouvelle.

\section{AUTEUR}

\section{SÉVERINE ABHERVÉ}

Docteur de l'Université Paris 1, Panthéon-Sorbonne 